\title{
The Large Synoptic Survey Telescope: A summary update on the scientific potential for pulsating star research
}

\author{
Melissa L. Graham ${ }^{1, \star}$ \\ ${ }^{1}$ Department of Astronomy, University of Washington, Box 351580, U.W., Seattle WA 98195, USA
}

\begin{abstract}
The Large Synoptic Survey Telescope (LSST) will provide hundreds of deep images of the southern sky over its 10 year duration, enabling variability studies for an unprecedentedly large and unbiased population of objects. In this proceeding paper I will cover the aspects of the LSST's survey and data products that are most relevant to the study of stellar pulsations (Sect. 1), and provide a directory of pertinent materials for further information. I will also summarize the anticipated variable star sample sizes from the LSST, and highlight recent research from several members of the scientific community which evaluates the scientific potential of the LSST's data products with respect to pulsating stars (Sect. 2).
\end{abstract}

\section{The Large Synoptic Survey Telescope: An overview}

The Large Synoptic Survey Telescope (LSST) is currently under construction on Cerro Pachón in Chile. With an 8.4 meter primary mirror, a 3.5 degree radius field of view, and a 3.2 Gigapixel camera of 0.2 arcsecond pixels, the LSST will image the full sky area of 18000 square degrees hundreds of times over its 10 year main "wide-fast-deep" survey (see Table 1 for a link to the project website). These repeated visits allow for the discovery and monitoring of moving objects such as stars with proper motion, and transient and recurrent variables such as pulsating stars. Deep stacks of all images will enable the detection and characterization of faint objects, and the wide unbiased survey area will provide sample populations of unprecedented number. A description of the full range of science drivers and how they have influenced the project's design specifications for the telescope and survey can be found in Ivezić et al. ([1]), and a breakdown of these specifications can be found in the LSST Science Requirements Document ([2]). The LSST Science Book ([3]), prepared by the scientific community, covers many of the anticipated goals in observational astronomy that could be accomplished by the LSST. The scientific community has also collectively produced a comprehensive white paper that analyzes observing strategies for the wide-fast-deep survey and makes recommendations for optimizing a variety of science goals ([4]). Aside from the wide-fast-deep survey, the LSST plans to incorporate "deep drilling fields" (DDF) and "mini-surveys" to attain additional science goals. White papers from the scientific community proposing DDFs were generated in 2011 and four extragalactic fields were chosen (see Table 1 for a link to DDF information). A call for additional fields, cadence proposals for the selected DDFs, and mini-surveys is expected to be released in the next year.

${ }^{\star}$ mlg3k@uw.edu 
Table 1. Useful links to information about the LSST project and associated science initiatives.

\begin{tabular}{|c|c|}
\hline LSST Resource Description & Weblink URL \\
\hline LSST Project Website & http://project.lsst.org \\
\hline Science Drivers to Reference Design [1] & http://arxiv.org/abs/0805.2366 \\
\hline The LSST Data Management System [5] & http://dm.1sst.org/ \\
\hline LSST Science Requirements (LPM-17, [2]) & $\begin{array}{l}\text { http://arxiv.org/abs/1512.07914 } \\
\text { http://ls.st/LPM-17 }\end{array}$ \\
\hline LSST Data Products Definitions (LSE-163, [6]) & http://ls.st/LSE-163 \\
\hline LSST DM Applications Design (LDM-151, [7]) & http://1s.st/LDM- 151 \\
\hline LSST Github Repositories & http://github.com/lsst \\
\hline LSST Software User Guide (The Stack) & http://pipelines.lsst.io \\
\hline LSST Community Forums & http://community.lsst.org \\
\hline LSST Science Collaborations & http://lsstcorporation.org/science-collaborations \\
\hline The LSST Science Book [3] & http://arxiv.org/abs/0912.0201 \\
\hline & http://lsst.org/scientists/scibook \\
\hline White Paper: Observing Strategy [4] & http://github.com/LSSTScienceCollaborations/ObservingStrategy \\
\hline White Papers: Deep Drilling Fields & http://project.lsst.org/content/whitepapers 32012 \\
\hline LSST Data Science Fellowship Program & http://ciera.northwestern.edu/Education/LSSTC_DSFPOverview.php \\
\hline Review: Maximizing Science in the Era of LSST & http://noao.edu/meetings/lsst-oir-study \\
\hline
\end{tabular}

An overview of the LSST data management system can be found in Jurić et al. ([5]). There are two main data processing pipelines: the Alert Production (AP) and the Data Release Production (DRP). The AP will run in real time, generating and searching difference images and releasing alerts on variable objects within 60 seconds of each observation. Processed images and catalogs from the DRP will be released to the community on an approximately yearly basis. Additional user-defined data processing for science goals not achievable with these products will be developed through the LSST Science Platform. All of the code for processing data from the LSST - collectively referred to as "The Stack" - is open-source and available online through GitHub (see Table 1). Documentation and user guides are included in the hope that the LSST end-users will find the Stack helpful when developing their own added-value processing and analysis through the LSST Science Platform.

In-depth information about the products that will be provided by the LSST data management system, and the processing methods that will be used to generate them, are described in the LSST Data Products Definitions Document ([6]) and the LSST DM Applications Design Document ([7]), respectively. The data products that will be of particular interest to the stellar pulsations community include:

- Co-added Image Photometry - Sources will be detected, deblended, measured, and calibrated for all coadd images; these measurements include aperture fluxes. Types of co-adds will include "deep" and "best-seeing", unless they're shown to be one and the same, and "short-period" co-adds, e.g., over one to several years, in order to identify faint long-period variables.

- Single Visit Photometry - Sources will be detected, deblended, measured, and calibrated for all single visit images and difference images ${ }^{1}$. Forced photometry on single visit and difference images will be performed at the location of all difference-image sources detected (for AP, this is limited to detections in the previous 12 months; for DRP, there is no limit). All newly-detected difference image sources will have forced photometry generated from the past 30 days of difference images.

- Variability Characterization - The exact quantities are to be determined, but the object catalogs will contain commonly-used values that represent variability, such as periodic features extracted from the light-curve based on generalized Lomb-Scargle (similar to, e.g., Table 4 of [8]).

\footnotetext{
${ }^{1} \mathrm{~A}$ difference image is a single visit image subtracted from a previously-generated template image.
} 
The author welcomes correspondence with the scientific community and future users regarding additional catalog or image products that would enable science projects with LSST data ${ }^{2}$, and/or questions about the current plans for the LSST data management system and its products.

\section{The LSST and pulsating star research}

Preparations for science with the LSST are occurring mainly within the established Science Collaborations (SC). The two SC most relevant to pulsating star research are Transients and Variable Stars ${ }^{3}$ and Stars, Milky Way, and Local Volume ${ }^{4}$. The SC work together through telephone and in-person meetings, "hack-a-thons", and online community forums, and collaborative efforts such as writing the white paper on Observing Strategy, building science roadmaps, and running data challenges are also underway (see Table 1 for links to the forums and the white paper). Eligible members of the scientific community are welcome to submit applications for SC membership (see websites for details; membership requirements vary by SC). Aside from the SC, reviews such as "Maximizing Science in the Era of LSST: A Community-based Study of Needed US OIR Capabilities" are assessing whether the current and planned resources will be able to support LSST-related science, and a fellowship program for students to learn data science techniques that will be applicable to the LSST data products has been established (web links to the review and the fellowship program are in Table 1).

The Science Book ([3]), especially chapters 6 (Stellar Populations), 7 (Milky Way and Local Volume Structure), and 8 (The Transient and Variable Universe), detail how the LSST will advance pulsating star research by providing a larger sample at fainter magnitudes and longer timescales. The predicted yields are $\sim 135 \times 10^{6}$ variable stars, of which $\sim 59 \times 10^{6}$ are pulsators ([3]). The Science Book describes how the main science goals to be enabled by these populations include:

- RR Lyrae: The LSST will provide $~ 10^{5}$ stars in the Magellanic Clouds with well mapped stellar populations for studying relations in brightness, period, age, and metallicity.

- Cepheid Variables: The larger samples provided by the LSST can address intrinsic variation, improve calibration, and provide distances for more Type Ia supernova host galaxies for cosmological studies.

- White Dwarfs: The LSST's photometric precision can identify new candidate pulsating white dwarfs and provide the ability to find and map (new) instability strips. The LSST's light curve characterizations can constrain, e.g., core composition and physics.

- Asymptotic Giant Branch Long-Period Variables: The large sample from LSST can map out the structure of the Milky Way and nearby galaxies in stellar mass, age, and dust production. The LSST's light curve characterization can constrain internal physics and nucleosynthesis.

Also relevant to RR Lyrae science, Sesar et al. ([9]) show that as with the Sloan Digital Sky Survey (SDSS) data, the multi-band aspect of the LSST survey will improve the capability for photometric classification of RR Lyrae stars, which can be difficult to distinguish from, e.g., eclipsing binaries with single-filter observations.

The Observing Strategy white paper on the wide-fast-deep survey has several sections that are relevant to the LSST science capability for pulsating variable stars. The following paragraph is not a complete summary of all pulsating-star science addressed in the Observing Strategy white paper, but intended to highlight and exemplify the type of analysis being done. Section 5.2.1 describes

\footnotetext{
${ }^{2}$ E.g., see this call for science community feedback on DDF processing and data products: https://community.lsst. org/t/deep-drilling-fields-and-data-management/1115/1.

${ }^{3}$ https://tvs.science.lsst.org/

${ }^{4}$ https://milkyway.science. lsst.org/
} 
metrics for optimizing LSST observations for Cepheid star science, which are also presented in Lund et al. ([10]). Two example metrics are: (1) periodogram purity function metric, which represents the fraction of power leaked away from the true frequency due to, e.g., sampling cadence and photometric precision, and (2) the field star counts metric, which evaluates the number of LSST filters in which a star would be detected, as a function of stellar mass and distance, for a given observing strategy. This section concludes that the Cepheid stars will be well characterized by the default cadence, but that a more rapid cadence would result in faster discovery and classification, enabling more science in the early years of the survey. Another example relevant to pulsating star research is Section 5.3, written by Keaton Bell, which presents the VarDepth metric: the magnitude limit for which $90 \%$ of pulsators with a $1 \%$ root-mean-square variability will be detected (i.e., a ZZ Ceti star). This work shows that $<100 \mathrm{ZZ} \mathrm{Cetis} \mathrm{would} \mathrm{be} \mathrm{discovered} \mathrm{and} \mathrm{characterized} \mathrm{in} u$-band with the default observing strategy, lower than the sample size of 1000 that would have significant scientific impact. See also the work of Bell et al. ([11]) for a prediction of the number of outbursting cool white dwarf pulsating stars that will be found with LSST. Section 5.5 references the work of Oluseyi et al. ([12]), who predict that at 10 years, the LSST will recover $50 \%$ of the RR Lyrae stars at $600 \mathrm{kpc}$, which is sufficient for the LSST science goals of finding Local Group halo streams and dwarf satellites, estimating their metallicities and placing constraints on formation scenarios. Two of the main conclusions from these and other studies included in the Observing Strategy white paper are that there are two types of cadences that are detrimental to the discovery and period recovery of pulsating stars: exactly uniform visit spacing and extremely non-uniform visit spacing (i.e., concentrating all visits of a field into a few years). As neither of those are likely to happen, the field of periodic variable stars appears poised to meet its LSST science goals for the wide-fast-deep main survey.

As a final note, the recent publication by VanderPlas \& Ivezić ([13]), titled "Periodograms for Multi-band Astronomical Time Series", builds on the RR Lyrae predictions of Oluseyi et al. ([12]) with a new technique that models the light curve as a base function common to all bands, plus a residual for each filter. VanderPlas \& Ivezić show that their new method outperforms other options, especially for the faintest periodic variable stars: at 5 years, the LSST can reach $100 \%$ completeness for RR Lyrae period measurements down to $g=24.5$ magnitudes, and at 1 year, the LSST can be 50\% complete for RR Lyrae to $g \sim 23.8$ magnitudes ([13]). The latter is a remarkable increase in limiting magnitude by $>1.5$ magnitudes over previous estimates, increasing the $50 \%$ completeness distance from 100 to $\sim 250 \mathrm{kpc}$, so far into new territory in the Milky Way's halo that the expected number counts are unknown.

\section{Summary}

At the time of writing, both the hardware and software for the LSST are under active construction, and the processes and products of the LSST data management system are becoming solidly defined. This proceedings document has outlined some of the anticipated data products that will be most relevant to the stellar pulsations community. All readers are welcome to contact the author with feedback regarding additional products that would enable science (e.g., light-curve variability parameters, specialized co-adds). This document has also highlighted the significant contributions made by members of the scientific community towards optimizing the LSST's main survey strategy and maximizing science return in the field of pulsating stars. In particular, recently developed analysis tools show a promising ability to enhance the science capability of LSST data products after just one year of the survey, building larger samples of pulsating stars and unlocking LSST science goals earlier than expected. 
Acknowledgments: This material is based upon work supported in part by the National Science Foundation through Cooperative Agreement 1258333 managed by the Association of Universities for Research in Astronomy (AURA), and the Department of Energy under Contract No. DE-AC02-76SF00515 with the SLAC National Accelerator Laboratory. Additional LSST funding comes from private donations, grants to universities, and in-kind support from LSSTC Institutional Members.

I thank Željko Ivezić, Keaton Bell, and Paula Szkody for helpful conversations and for their contributions to the content of my oral presentation at the meeting, and I thank the conference organizing committee for extending the invitation to speak and for covering my accommodations and registration. I very much appreciate the many good conversations I had with other attendees throughout the meeting, and I'd like to extend a special thanks to the local organizers for arranging the unique opportunity to visit the ALMA site.

\section{References}

[1] Ž. Ivezić, J.A. Tyson, B. Abel, E. Acosta, R. Allsman, Y. AlSayyad, S.F. Anderson, J. Andrew, R. Angel, G. Angeli et al., ArXiv e-prints (2008), 0805.2366

[2] Ž. Ivezić et al., LSST Science Requirements Document, LSST Project Management LPM-17 (2011), http://1s.st/srd

[3] LSST Science Collaboration, P.A. Abell, J. Allison, S.F. Anderson, J.R. Andrew, J.R.P. Angel, L. Armus, D. Arnett, S.J. Asztalos, T.S. Axelrod et al., ArXiv e-prints (2009), 0912 .0201

[4] P. Marshall et al., Science-Driven Optimization of the LSST Observing Strategy, in preparation (2017), https://github.com/LSSTScienceCollaborations/ObservingStrategy

[5] M. Jurić, J. Kantor, K. Lim, R.H. Lupton, G. Dubois-Felsmann, T. Jenness, T.S. Axelrod, J. Aleksić, R.A. Allsman, Y. AlSayyad et al., ArXiv e-prints (2015), 1512 .07914

[6] M. Jurić et al., LSST Data Products Definition Document, LSST Data Management LSE-163 (2016), http://ls.st/LSE-163

[7] M. Jurić et al., LSST Data Management Applications Design, LSST Data Management LDM151 (2013), http://ls.st/LDM- 151

[8] J.W. Richards, D.L. Starr, N.R. Butler, J.S. Bloom, J.M. Brewer, A. Crellin-Quick, J. Higgins, R. Kennedy, M. Rischard, ApJ 733, 10 (2011)

[9] B. Sesar, Ž. Ivezić, S.H. Grammer, D.P. Morgan, A.C. Becker, M. Jurić, N. De Lee, J. Annis, T.C. Beers, X. Fan et al., ApJ 708, 717 (2010)

[10] M.B. Lund, R.J. Siverd, J.A. Pepper, K.G. Stassun, PASP 128, 025002 (2016)

[11] K.J. Bell, J.J. Hermes, M.H. Montgomery, D.E. Winget, N.P. Gentile Fusillo, R. Raddi, B.T. Gänsicke, The First Six Outbursting Cool DA White Dwarf Pulsators, in Astronomical Society of the Pacific Conference Series, edited by P.E. Tremblay, B. Gaensicke, T. Marsh (2017), Vol. 509 of Astronomical Society of the Pacific Conference Series, p. 303

[12] H.M. Oluseyi, A.C. Becker, C. Culliton, M. Furqan, K.L. Hoadley, P. Regencia, A.J. Wells, Ž. Ivezic, R.L. Jones, K.S. Krughoff et al., AJ 144, 9 (2012)

[13] J.T. VanderPlas, Ž. Ivezić, ApJ 812, 18 (2015) 